\title{
INTEGRATED AND CUSTOMER-ORIENTED MATERIAL AND PROCESS SELECTION BY SENSORY MULTI-CRITERIA DECISION-MAKING
}

\author{
Schneberger, Jan-Henrik; Kaspar, Jerome; Vielhaber, Michael \\ Saarland University, Institute of Engineering Design
}

\begin{abstract}
Today, product success more than ever depends on the satisfaction of consumer needs. Besides, manufacturers need to shorten development cycles and accelerate product release in order to stay competitive. This is achieved by applying customer-oriented methods allowing for fast and reliable acting. During the early phase of product development, requirements acquisition is crucial for later success of products, since specifications are most influenceable at this point. Referring to the decisive concept generation phase, material and production definition is difficult due to the highly complex interrelations between material properties, production process capabilities, and resulting product characteristics. Especially in the context of lightweight design, concurrent material and processing technology selection must be considered due to its various possible interfaces.

Thus, this contribution outlines an integrated approach towards an enhanced material and process related, customer-oriented requirements acquisition during the early phase upstream of product detailing. Here, conventional multi-criteria decision-making and tactile recognition are employed in an agile transformation model.
\end{abstract}

Keywords: Decision making, Requirements, Integrated product development, Customer-oriented material selection

Contact:

Schneberger, Jan-Henrik

Saarland University

Institute of Engineering Design

Germany

schneberger@1kt.uni-saarland.de

Cite this article: Schneberger, J.-H., Kaspar, J., Vielhaber, M. (2019) 'Integrated and Customer-Oriented Material and Process Selection by Sensory Multi-Criteria Decision-Making', in Proceedings of the 22nd International Conference on Engineering Design (ICED19), Delft, The Netherlands, 5-8 August 2019. DOI:10.1017/dsi.2019.123 


\section{INTRODUCTION AND MOTIVATION}

The product development process is characterized by several multilateral aspects concerning different fields of interest. First, ever-shorter development cycles including fast and precise iterations are aspired in order to stay in touch with the latest state of the art in widely relevant branches (e.g., automotive industry, consumer electronics, and artificial intelligence) from both a customer and entrepreneur point of view. Second, products need to be developed economically in order to provide maximum financial benefit, going along with the best-possible customer satisfaction.

Therefore, a versatile requirement definition, which is the first and foremost stage in product development, needs to be executed carefully, as it provides the most important document for further proceeding; the requirements list (Pahl et al., 2007). Precisely looking from the discerningly changing customers' side, products more and more need to be unique by means of individualization and gaining prestige status. Consequently, seriously integrating the customer already during product creation is nowadays unavoidable for OEMs seeking to be successful. This goes along inherently with a simultaneous view on the advantageous production aspects interacting and correlating with the demanded fast and cost-efficient development vision. In doing so, bilateral requirements can be considerably earlier and more purposeful identified (Dick et al., 2017) and brought into a context of mutual influence and interference, affecting product and production process characteristics. However, current practice does not sufficiently involve the customer in an integrated material and process selection process, and thus wasting potential for customer satisfaction and development process acceleration.

On this basis, the present contribution constitutes an extended procedural approach towards an integrated material and process selection aiming at the initial stage of requirements specification, additionally including a comprising transformation procedure to gain hard requirements from vaguely formulated customer needs. Focusing on coherences between individual customer perception and material, both promising and devastating interrelations are brought into light and being furtherly connected to the production process selection by reconciliation with the available technological performance spectrum of individual processes.

\section{STATE OF THE ART IN LITERATURE AND PRACTICE}

The following chapter reviews the current state of the art in literature considering requirements engineering activities in common design and development process models, customer-oriented design approaches, as well as engineering practice in material and process selection.

\subsection{Requirements acquisition in conventional product development processes}

Common methodological approaches concerning product development provide an initial project and task planning phase, followed by product definition, conceptual design and embodiment design phases, e.g. proposed by Pahl et al. (2007), Ulrich and Eppinger (2008), or Ullman (2010). These approaches integrate requirements engineering in certain phases and consider different intensities, but not as the main constituent in focus. Requirements engineering activities, as depicted by Dick et al. (2017), are conducted individually up to a certain, not necessarily corresponding degree.

Thus, Pahl et al. (2007) initiate the development process with a specification phase that is the central element in product planning. The developer's main task is to gather and list customer requirements in a fully quantified way and "in close cooperation" (Pahl et al., 2007) with the customer. Ulrich and Eppinger (2008) propose a similar procedure to the aforementioned issue. They introduce an initial phase for the identification of demands, including direct consultation and interrogation of potential customers in oral interviews as well as tactile evaluation using physical prototypes of specific goods. Considering subsequent phases of the development process, Ulrich and Eppinger (2008) do not stipulate close integration of the customer in the design process, but indicate the necessity of the design and development team to align initial customer specification and conceptually achieved product characteristics consequently. Another approach presented by Ullman (2010) essentially indicates similarities with the previous methodological frameworks. During the initial product discovery, future customers and customer groups are identified in order to elaborate requirements that need to be fulfilled to fit the customer needs (Ullman, 2010). Simultaneously, competitors' products are evaluated in terms of requirements and quality accomplishment. Ullman (2010) recommends oral interviews of customers to gather requirements and furtherly trace them 
by mapping requirements and product characteristics utilizing the standard QFD by Akao (1990). Although the customer is integrated in the early phase of product discovery, supplementary interactions between developer and customer in successive stages are again not provided.

\subsection{Customer-oriented and innovation-driven requirements specification}

Customer-oriented design is one of the core strategies in past and today's economic environment and market situation (Akao, 1990; Dagher and Petiot, 2007). Due to the immense variety of products available, satisfying customer needs and requirements is crucial for the design of products and their success on the market (Dick et al., 2017). By executing requirements engineering activities, for example, developers are able to elicit, document and trace requirements alongside the whole development process (Dick et al., 2017). One of the well-known techniques is featured by the QFD (Akao, 1990), used to determine customer requirements considering quality aspects and subsequently map those requirements with qualitydetermining product characteristics. In a first step, customer requirements are gathered by a thorough analysis of customer input - weather in oral interviews or by studying complaint (Akao, 1990) - in order to transfer a customer demand into engineering terms afterwards. This principle is systematically executed and kept in a quality chart being continuously expressed by the House of Quality method as proposed by Hauser and Clausing (1988). QFD is primarily based on customer demands and requirements, whereas potential customers themselves do not necessarily need to be integrated into the development process, which may be harmful. Requirements acquisition can also be done by project and design teams, whilst this may lead to incorrect assumptions (Pohlmeyer, 2012). Utilizing the House of Quality, QFD can furtherly be implemented to determine quality relevant aspects in production and service leading to a holistic product lifecycle engineering. Thus, related work on this topic integrates fuzzy QFD, amongst others, for the determination of market and manufacturing strategies based on a customer-oriented development often combined with multi-criteria decision-making (Jia et al., 2011; Dat et al., 2015; Lima-Junior and Carpinetti, 2016).

A more open and fuzzy determination of requirements is given by the Kano model, which differentiates requirements and needs between "must-be requirements", "one-dimensional requirements" and "attractive requirements" (Pohlmeyer, 2012). Data from the Kano model predominantly appear qualitative, whereas quantified declarations cannot be given without further investigation. Deficiencies of the Kano model as well as the combination with QFD have been analyzed and assessed in the literature, for example, He et al. (2017). In order to gather information and trustworthy requirements without having individuals or a group of customers to consult, personas - originating from human-computer-interface design - appear to be suitable. Fictional personas represent individuals or even larger groups of customers (Pohlmeyer, 2012; Sim and Brouse, 2014) and provide developers with an opportunity to layout and critically assess potential target markets, which either cannot be accessed immediately or only with disproportionate effort. Considering personas as a tool, quite similar to scenario techniques in a narrow event horizon, the results drawn from persona definition are likely to be corrupted because of individual preferences assigned to the artificial customer representation, actually considered neutral and unbiased as emphasized by Janhager and Hagman (2007). Further approaches can be identified within the modern Design Thinking, which facilitates user-centered design in a partly workshop-based development process. However, these techniques are not yet really established in the industrial environment.

\subsection{Material and process selection approaches}

To date, a systematic procedure for selecting materials and with it processes to manufacture the actual component is essential within a consistent product development, ideally right from the beginning. Thus, several approaches describe this complex and multi-attribute decision-making issue from the past to the present. Ashby (2011), who introduces four main steps up to the final material selection in his internationally accepted standard reference work, outlines one of these. Herein, the translation and gathering of material-related requirements serves as the starting point for the subsequent screening, ranking, and final selection covered by a continuous identification and refined specification of elementary component-based demands (function, constraints, objectives, and free variables). Through the years, this simple and clear material selection strategy was developed into a powerful computer-based material expert system (Cambridge Engineering Selector by Granta Design Ltd. (2015)) enabling a transparent visualization of eligible materials in shape of so-called more-dimensional material property charts. Apart from this primarily material-driven approach, various scientific contributions deal with a combined selection strategy for materials and processes (Ashby et al., 2004), materials and shapes (Weaver and 
Ashby, 1997; Pasini, 2007), or - as already mentioned - more customer-centered aspects (Karana, 2009). However, and even the topic of simultaneous engineering tends to be fully treated in the past (e.g., DfMA approach by Boothroyd (1994)), a more holistic consideration of all three dimensions (material, design, and process) including cross-component aspects and challenges is exclusively framed by Kaspar and Vielhaber (2016; 2018) and Kaspar et al. (2018). In this context, the authors examine a systemic analysis and assessment of technical, economic and ecological benefits regarding a combined component and joint section design.

\subsection{Demands on an integrated, customer-oriented material and process selection}

A brief review of the current state of the art in literature considering requirements engineering and its implications on an integrated material and process selection shows that there is still a lack of explicit methods and procedures ensuring sufficient considerations on interrelations between the disciplines. Moreover, current requirement sources may be inaccurate due to inconsistent elicitation and interpretation of customer needs - in some circumstances not even gathered from the real customer milieu. This leads to reliability issues concerning the determination of design characteristics desired by the customer as well as materials and processes in the early phase of product development, eventually requiring an upstream relocation of planning efforts in the production process, i.e. a shift towards the later stages of development. As stated above, this situation must be avoided in order to facilitate short iterations and an economic development of products later on.

Therefore, the herein presented approach promotes a direct interaction with the customer considering a structured procedure to determine superior needs and requirements regarding the three disciplines, in an integrated way. Design as well as material requirements are treated coherently, which allows for the elimination of primary inconveniences. A comprehensive assessment of materials and processes supplies information on the feasibility of manufacturing and joining operations simultaneously. As a further step, cross-correlations of design and manufacturing processes enable developers to determine and, if required, eliminate possible and incongruous matches.

\section{CUSTOMER-ORIENTED MATERIAL \& PROCESS SELECTION APPROACH}

To take advantage of discipline-specific benefits bypassing the gap between customer domain and explicit customer-integrating material and process definition, this section introduces the customer-oriented, integrated material and process selection approach.

\subsection{Levels of customer integration in the definition procedure}

The creation of an approach, irrespective of which target is pursued, requires the definition of preconditions and considered actors. Without these aspects clearly outlined, the approach cannot fulfill its desired effect and may fail to deliver results in a proposed manner.

The procedure outlined in this contribution supposes the developer to be a professional with an intense and deep knowledge of technical systems and technological circumstances, especially regarding mutual influences between material selection and process characteristics. Thus, the professional is responsible for adhering to the structured procedure provided. In order to satisfy a customer-oriented development, on the one hand, the developer is supposed to stay neutral and unprejudiced against any data collected during the specification process. On the other hand, preconditions regarding the customer are estimated diametrical. Moreover, the authors assume that the herein presented procedure shall be tailored to fit nonprofessional customers by generating sophisticated support in elicitation and refinement of needs and demands. Professional customers, those with an educational, technical or technological background, are expected to be able to specify their demands sufficiently quantified. This results in the aforementioned anticipation of a layman customer or client.

The procedure is designed to incorporate three stages according to different levels of required precision (exemplarily referring to the steps in material selection proposed by Ashby et al. (2011)), focusing on fully defining boundary conditions of the classification of material class, subclass, and type. This contribution, however, transfers the three stages into individual perception levels, simultaneously representing different levels of specificity. The customer is associated to all three levels, whilst its influence on the different aspects of material and process selection varies in scope and significance. Owing to the required education and routine in technological issues, the customer yields significantly more influence on the selection of materials, since the interaction with a technical product or system mandatorily presumes to interact with a 
physical interface that involves material considerably more than the underlying manufacturing process. Ashby and Johnson (2014) have already deduced evidence for the importance of materials as a physical interface. As opposed to this, the developer as an educated and trained professional will have more influence on the determination of required process characteristics. Nevertheless, and to fulfill the demands proclaimed by the approach of customer-oriented development, the customer is the main protagonist in a closed-loop feedback and adjustment cycle alongside the whole product development process, even exceeding material and process selection in the initial stages.

\subsection{Correlation of customer specifications, material and process characteristics}

The proposed procedure follows the principle of gradual condensation of information from a rather rough specification and humble validity into highly detailed, compulsory facts that form the target line. In this contribution, the QFD-inspired customer interrogation is initially focused on the visible area of a product, since non-visible parts' composition does not attract primal attention at first inspection.

Figure 1 shows the overall procedure from requirements elicitation towards condensation.

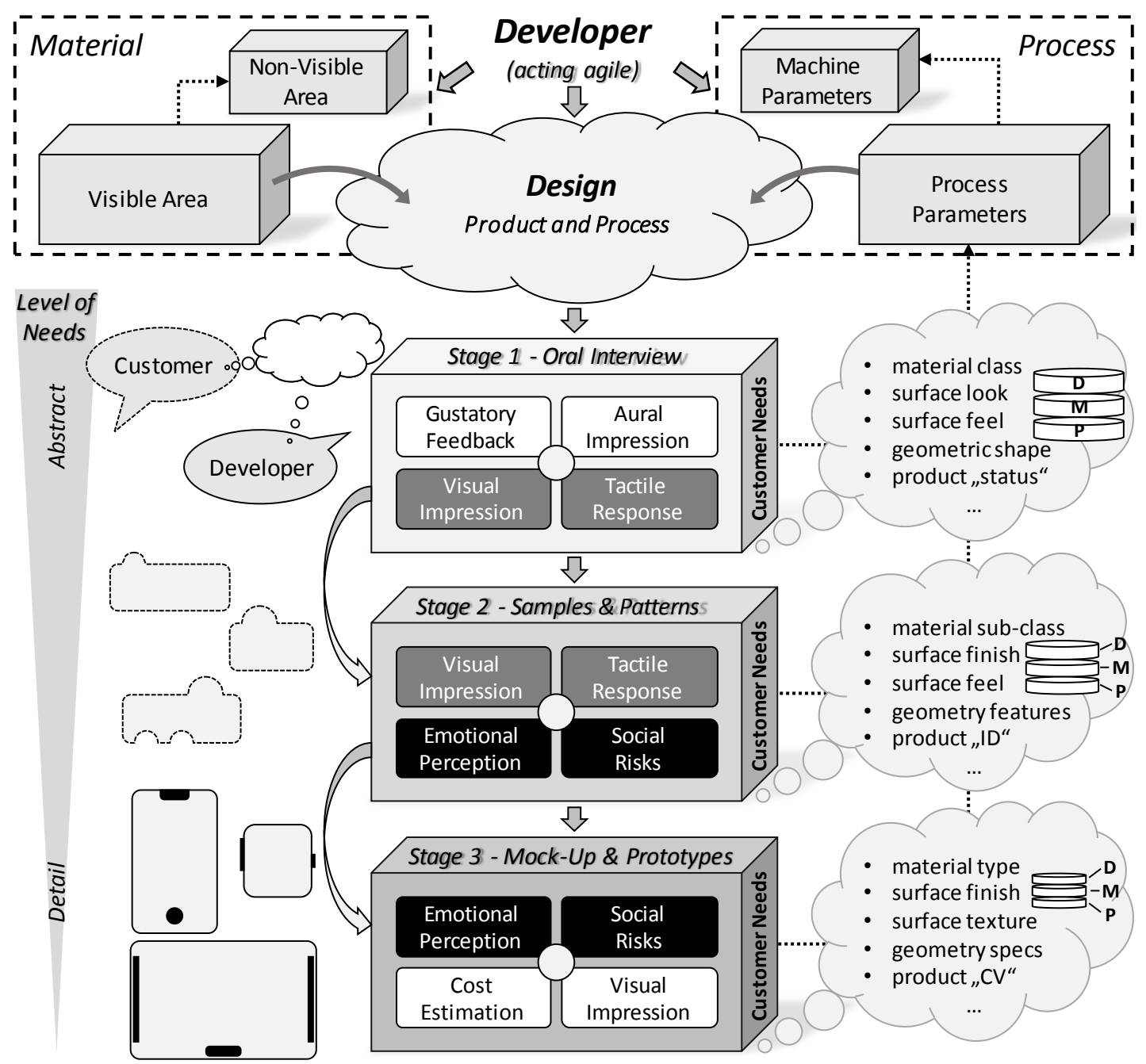

Figure 1. Sequential stage-model for elicitation and gradual refinement of requirements regarding material and process selection

The proposed procedure is applied in order to determine and merge required material and process properties into a comprehensive product description, which later results in appropriate concept as well as embodiment designs. Herein, material and process correlations are utilized as guidelines and boundary conditions, determining achievable solutions in clearly delimited solution spaces. However, influence from the customer side is supposed to be decreasing during the transition from material towards processes due to the rising specificity of required knowledge and complexity.

The first stage consists of an oral interview in which the developer follows a catalogue of possible questions concerning the product or system to be developed. Conceivable questions are, for example, 
"How big should the object be?", "Should the object be shiny or dull?" or "Do you feel like the object is made from metal, plastics, or wood?". On this level, the customer's perception is evaluated focusing on the basic human senses comprising the gustatory (including olfactory perception), tactile, visual and aural senses. The sense-based attribution of materials has been proven for a common description rather than explicit selection in terms of a customer-centered development by Karana (2009) as well as Ashby and Johnson (2014). The results of the first stage are expected to be coarse. Insufficient precision may occur due to the comparatively early stage of development. Regarding the view of the customer, it becomes obvious that production process selection cannot be conducted reliably for the lack of credible criteria (customers' knowledge regarding production must be assumed considerably low). Nevertheless, the first stage is expected to output information on fundamentally possible material classes, ideally reduced to only one specific class without any room for interpretation. By setting the material class at this point, further information can be processed in the downstream stages utilizing, for example, databases promoted by Ashby (2011), to define actual material subclasses hereafter. The identification of customer needs and demands is supported by the invitation to imagine, dream up and, for example, mould the ideal product shape.

Moving on to the second stage, the sensory view on the product is narrowed by focusing on visual and tactile responses, whereas overall consideration is extended by emotional and social desires. The concept of emotions in engineering is based on the urge to include non-physiological needs in product creation, as proposed by Kett and Wartzack (2016), and owing to the fact that products are often used to express individuality, status, and prestige. By including the emotional and social factors, this contribution assures a comprising view on both tangible and intangible aspects of products in general. The customer contributes to the second stage by revealing detailed information on demands, which are initially unquantified. Those arise from different causes, for example, personal and emotional motivation. Aspects, which can be addressed on this level, exceed the initial, shallow coverage and enrich the determination process by providing information on which ecological and economic boundary conditions have to be observed in order to obtain acceptance from the customer, or - thinking further - society, for instance. Regarding design, the authors follow the recommendation involved in QFD to use patterns and approval samples to elicit customer needs, as schematically indicated in Figure 1. The second stage therefore incorporates the use of those means, furthermore enriched by IT-databases capturing coherence and occurrence in products being already available on the market, which fosters early reconciliation of customer needs and material-processrelations. Here, process-related characteristics particularly include dependencies determining future surface constitution, such as achievable edge sharpness or bending radii, surface roughness, and possible heat treatment.

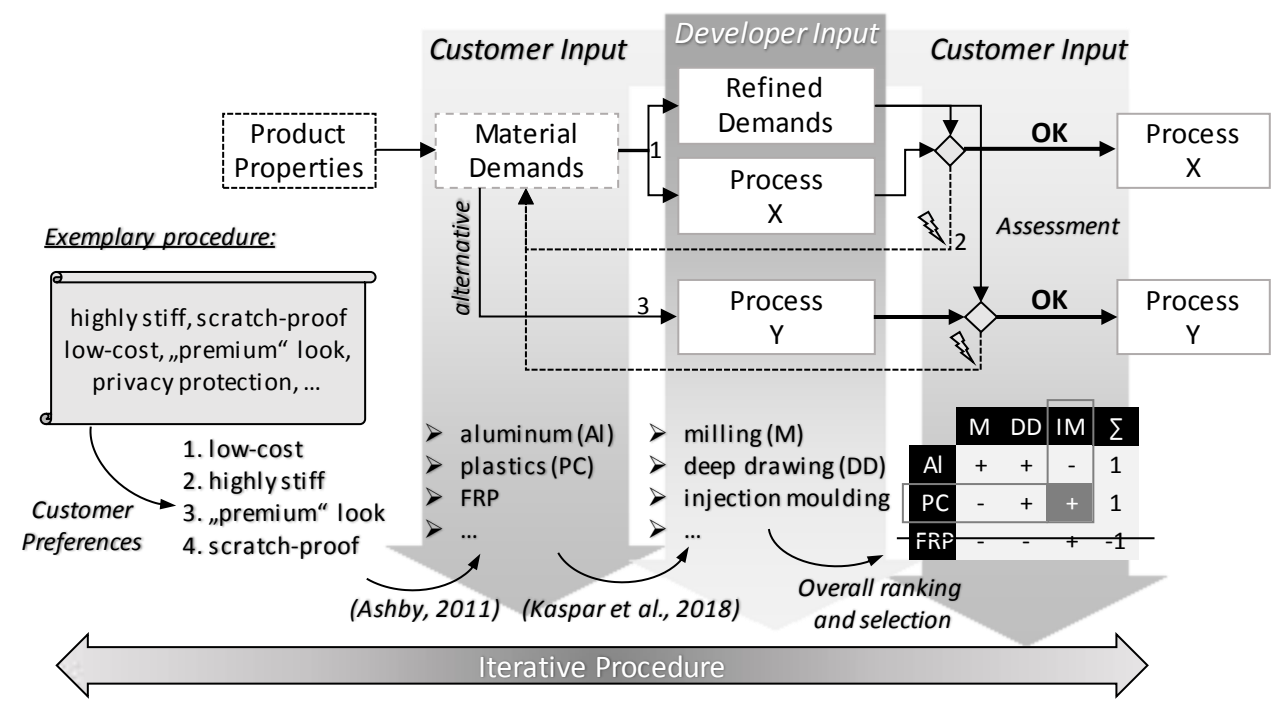

Figure 2. Matching-routine for suitability-assessment of material-process-couples

The third stage is supposed to target highly precise requirements concerning both material and manufacturing process characteristics. As in the previous stages, the customer is mostly responsible for material specification, whereas the developer now yields significantly more influence on material type selection as well as production process assessment. Physical prototypes and mock-ups support the procedure to guarantee the highest possible validity of specifications. The essential outcomes of this stage 
are technically, economically and ecologically most valuable material types and processing requirements (e.g., information on lot size) to determine economic limitations. Figure 2 depicts the framework, in which the developer acts as the facilitator. A short example illustrates the procedure by giving three suitable materials and processes for a given set of customer requirements, which is part of the application example presented in more detail in section 4.

The central element in the correlation approach is the so-called correlation-map (Kaspar et al., 2018), in which process characteristics and material attributes are confronted with each other, showing potential intersection in their mutual influence. Peculiar emphasis lies on the micro-cycle (1-2-3) that is performed during the clarification. Whilst the customer gives suggestions on material demands, suitable processes and required refinements are applied acting as the basis for further investigation. Following the herein presented routine, the customer is consulted after every definition step (including a detailed explanation of process parameters involved for the customer). Moreover, information on suitable materials and processes is given. This closed-loop feedback cycle is mandatory and must not be omitted in order to insure continuous information flow. Since necessary information on process parameters may be of various types, Table 1 exemplarily summarizes different kinds of information to be provided to the customer during the individual stages.

Table 1. Exemplary information scope and information update triggers

\begin{tabular}{|c|c|c|}
\hline & Information on process and material & Necessities for information update \\
\hline Stage 1 & $\begin{array}{ll}\text { - } & \text { Basic process information } \\
\text { - } & \text { Available material spectrum } \\
\text { - } & \text { Standardization degree of process }\end{array}$ & $\begin{array}{l}\text { - } \quad \text { Change in material class } \\
\text { - } \quad \text { Change in geometric dimension } \\
\text { - } \quad \text { Change in sustainability demands }\end{array}$ \\
\hline Stage 2 & $\begin{array}{ll}\text { - } & \text { Available material subclasses } \\
\text { - } & \text { Achievable process precision } \\
\text { - } & \text { Achievable repetition precision } \\
\text { - } & \text { Manufacturability of geometry }\end{array}$ & $\begin{array}{l}\text { - } \quad \text { Change in material subclass } \\
\text { - } \quad \text { Change in surface quality demands } \\
\text { - } \quad \text { Change in required tolerances }\end{array}$ \\
\hline Stage 3 & $\begin{array}{ll}\text { - } & \text { Available material types } \\
\text { - } & \text { Economic/ecological parameters } \\
\text { - } & \text { Manufacturing system integration }\end{array}$ & $\begin{array}{ll}\text { - } & \text { Change in material type } \\
\text { - } & \text { Change in costs and lot sizes } \\
\text { - } & \text { Change in sustainability issues }\end{array}$ \\
\hline
\end{tabular}

Obligatory indications on what information to promote (e.g., legal demands, road traffic acts) cannot be given due to multiple influences. First, the customer must not be patronized by withholding information that is supposed to be inappropriate during the specification process; moreover, the customer may not be asked too much in terms of understanding and subject specific education. According to the anticipated preeducation of customers, a rough categorization may be conducted in order to narrow the spectrum of data that need to be revealed, while quantity and quality of feedback cannot be estimated sufficiently.

\section{EXAMPLE AND VALIDATION}

In order to validate the herein presented method, an example from the consumer goods environment is used. The aim was to find material and process characteristics for the main physical user interface of a notebook (palm rest and display cover), focusing on potential customers' perception and choice of materials to be further used making assertions concerning suitable processes and overall design. Thus, suggestion and feedback cycles were performed as desired in Table 1 and lead to the aspired gradual refinement, as shown in Figure 3.

During the first stage, the interviewees were asked about requirements that concern a notebook in general (e.g., price and dimensions of the device) as well as specific demands regarding the aforementioned areas. Several tactile requirements were discovered collaboratively and many of them with haptic and optical concerns. The transformation process (quantification, as indicated in Figure 3) from qualitative towards quantitative requirements was applied successfully, yielding reliable specifications in terms of device dimensions (e.g., display size, max. allowed height, surface roughness, and edge sharpness), look (e.g., color, surface glance, and emblems) and primary functional requirements (e.g., required service temperature, corrosion resistance, and smell). To enable a quicker and more precise specification process, the applied guidelines contained current standards regarding concepts and real dimensions for notebooks 
and similar mobile consumer goods. However, the social factor could not be addressed appropriately in this stage, due to the prominence of the devices in any milieu.

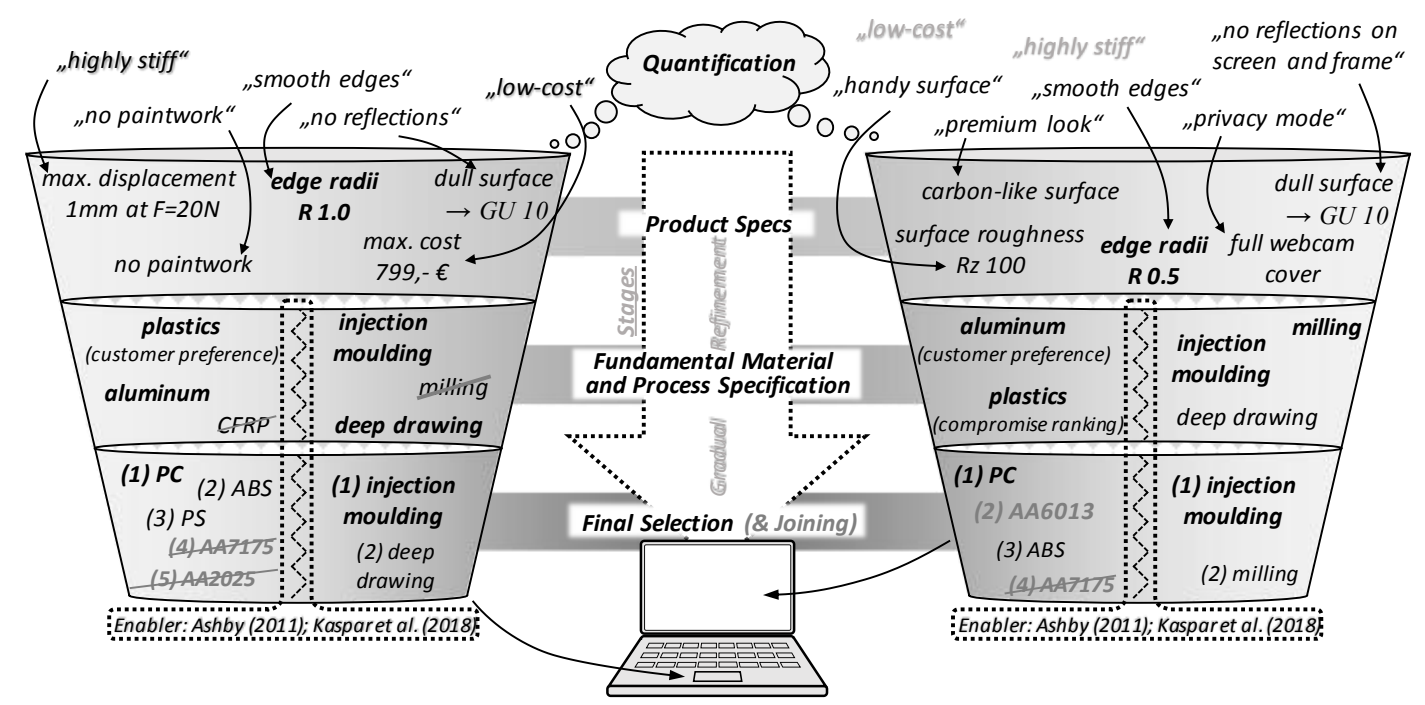

Figure 3. Illustrative application example (notebook with display cover and palm rest)

After the quantification of customer requirements, the interviewees were asked to give a ranking of their individual demands (exemplarily shown in Figure 2). The ranking provides a proper foundation for further investigation of the correlations between single requirements and processes. Hence, the interviewees had the opportunity to make a decision regarding suitable materials on their own (primarily without being given further information on technologically applicable solutions), which was afterwards supported by the provision of CES trade-off charts to give further background and prevent misassumptions. The information accessible in CES belongs to a rich repertoire to support the customer and lead towards a profound and satisfactory solution. Possibilities to encourage customers are diverse, so the authors do not claim completeness but focus on the methods and tools applied in this context.

The initial customer preferences for materials were highly influenced by currently available devices. However, due to the outcomes of different material and process related trade-off comparisons, CFRP was neglected due to its comparatively high price and complex manufacturing necessities, which contradicted the highest ranked requirement of "low-cost" components. According to the display cover, opinions were inconsistent. High stiffness and a low price are achievable with both aluminum and plastic parts, depending on which setup is chosen for the device. In conformity with prestige, however, some of the interviewees claimed that the component should be made from aluminum to achieve a premium look. Regarding processes, few of the technically affine interviewees took very profound decisions on the manufacturing of plastics, resulting in the initial preselection as depicted in Figure 3. Thus, a process mapping and provision of deeper insights was considered by nearly all of the interviewees, which resulted in the omission of milling for the production of the palm rest and keyboard compartment of the device due to material input and efficiency, and the inherent effects on waste volume and sustainability at last. Subsequently, the initial determination of suitable material classes and processing technologies was extended by the specification of material subclasses and types as well as matching or rather feasible manufacturing processes. According to the required stiffness and durability of both components, plastics could successfully be narrowed into, amongst others, polycarbonate (PC), acrylonitrile butadiene styrene (ABS) and polystyrene (PS). Regarding metallic materials, convenient aluminum alloys were found in, for example, AA2025, AA6013 or AA7175. As already indicated in Figure 1, the decreasing knowledge amongst the interviewers contributes to a growing significance of the developer in this stage. To narrow the solution space without disregarding the interviewees, the authors developed an overview chart of material subclasses and types, which would be suitable for this specific use-case. The authors solely used the initially defined requirements in order to stay unprejudiced and unbiased towards the interviewees. Confronting the customer with every single alternative does not contribute to a targeted selection of materials and processes due to the sheer variety of opportunities. The interviewees involved in this specific examination evaluated the procedure as appropriate, although technically educated individuals would have been more satisfied with even deeper insights into specific, mostly processual features. As a result, however, the materialprocess-combination was specified as PC, which is manufactured via injection moulding (IM), as 
referenced in Figures 2 and 3. Technologically worthy alternatives, such as deep drawing of plastics or aluminum, were collaboratively approved by both the customer and the developer.

Nevertheless, there are still aspects that could not be worked out sufficiently in this application case, for example, the perceived meaning of material choice from the customers' viewpoint. This is a highly complex issue in which multilateral demands and the assessment are confronted with the individuals' perception of quality and necessity, amongst others. Examinations regarding this issue will be treated in future contributions by the authors.

\section{DISCUSSION AND OUTLOOK}

Beginning with a state-of-the-art recapitulation concerning product development, material and process selection, as well as requirements acquisition, this contribution emphasizes specific imperfections regarding simultaneous material and process consideration. Moreover, it provides a customer-oriented extension to the agile requirements transformation process, which has been proposed by the authors earlier (Schneberger et al., 2018). This is achieved by the intense inclusion of potential or - as originally intended - real customers in the product development process, starting with a thorough requirements elicitation and documentation stage, provision of decision-making critical information during material and process selection, and continuous guidance of all parties involved in the early as well as consecutive stages. The approach presented in this contribution not only focuses on physiological demands brought forward by the customer, but also extends the view towards manifold non-physiological needs, which need to be addressed on the emotional and social level, massively influencing personal evolvement.

By promoting strict guidance by professionals, the non-professional customer is encouraged to formulate its demands unaffectedly, which requires an unbiased attitude of the developer towards the development target. The process and material selection is further characterized by the use of a correlation-map, which shows feasible positive and negative mutual influences. Configuration and application of the correlationmap are highly non-trivial due to the immense variety of materials and processes and their multidimensional interrelations. As an example, processing of aluminum necessarily depends on the respective alloy, since parameters vary significantly and have a huge impact on properties like formability or weldability. Therefore, future research activities on this topic need to intensify and concentrate on the composition of the correlation-map as well as on information and knowledge supply. A promising solution to support this process may be found in the implementation of artificial intelligence and machine learning. Large-scale systems can be analyzed strategically using elementary routines and set-based learning algorithms that provide gradual knowledge build-up, primarily fed by user input.

Regarding the form of the interviews, the definition of a holistic questionnaire or question-answercatalogue represents one of the most demanding tasks. In the application example, which can be found in section 4, the interviews were spontaneous, driven by the knowledge of the small-scope component. Giving an example on this: a customer asks for a certain design feature, without further specifying a material that shall be taken into consideration. The developers' task is then to transform these statements into quantified requirement. Thereby, the customer must be informed about all the materials and processes, which possibly satisfy the requirements, but also about the degree of fulfillment regarding any individual type. This includes potential alternatives, which might be less suitable in a specific field but more promising as a whole, as well as the status, which can be achieved regarding technological value, economic performance, and sustainability. This aspect might often be off scope, but regarding generally compatible manufacturing, the authors stress a more elaborate assessment. Based on this, the even more complex challenge is an advanced systemic, cross-component (joint section design) assessment in the end, combining all the customized parts into one functionally fitting and tailored product (Kaspar and Vielhaber, 2016).

\section{REFERENCES}

Akao, Y. (1990), “Quality Function Deployment”, Productivity Press, Cambridge.

Ashby, M. F., Brechet, Y. J. M., Cebon, D., and Salvo, L. (2004), "Selection strategies for materials and processes", Materials and Design, Vol. 25, No. 1, pp. 51-67. https://doi.org/10.1016/S0261-3069(03)00159-6.

Ashby, M. F. (2011), "Materials Selection in Mechanical Design". (4th ed.), Butterworth-Heinemann, Oxford. https://doi.org/10.1016/C2009-0-25539-5.

Ashby, M. F., and Johnson, K. (2014), "Materials and Design", (3rd ed.), Butterworth-Heinemann, Oxford. https://doi.org/10.1016/C2011-0-05518-7.

Boothroyd, G. (1994), "Product design for manufacture and assembly", Computer-Aided Design, Vol. 26 No. 7, pp. 505-520. https://doi.org/10.1016/0010-4485(94)90082-5. 
Dagher, A., and Petiot, J.-F. (2007), "Study of the correlations between user preferences and design factors: Application to cars front-end design", 16th International Conference on Engineering Design 2007, Paris, 28.31.07.2007, Design Society.

Dat, L. Q., Phuing, T. T., Kao, H.-P., Chou, S.-Y., and Nghia, P. V. (2015), “A new integrated fuzzy QFD approach for market segments evaluation and selection”, Applied Mathematical Modelling, Vol. 39 No. 13, pp. 36533665. https://doi.org/10.1016/j.apm.2014.11.051.

Dick, J., Hull, E., Jackson, K. (2017), “Requirements Engineering”, Springer International Publishing”, Cham, https://doi.org/10.1007/978-3-319-61073-3.

Granta Design Ltd. (2015), “CES Selector”, version 15.10.8. Cambridge. Available at: www.grantadesign.com.

Hauser, J. R., and Clausing, D. (1988), "The house of quality”, Harvard Business Review, pp. 63-73.

He, L., Song, W., Wu, Z., Xu, Z., Theng, M., and Ming, X. (2017), "Quantification and integration of an improved Kano model into QFD based on multi-population adaptive genetic algorithm”, Computer \& Industrial Engineering, Vol. 114, pp. 183-194. https://doi.org/10.1016/j.cie.2017.10.009.

Janhager, J., and Hagman, L. A. (2007), "Exploration of user-technical process scenario technique in practice", 16th International Conference on Engineering Design 2007, Paris, 28.-31.07.2007, Design Society.

Jia, G. Z., and Bai, M. (2011), “An approach for manufacturing strategy development on fuzzy-QFD”. Computer \& Industrial Engineering, Vol. 60 No. 3, pp. 445-454. https://doi.org/10.1016/j.cie.2010.07.003.

Karana, E. (2009), "Meaning driven materials selection in design education”, 17th International Conference on Engineering Design 2009, Vol. 10, Palo Alto, 24.-27.08.2009, Design Society, pp.69-80.

Kaspar, J., and Vielhaber, M. (2016), "Cross-component systematic approach for lightweight and material-oriented design”, NordDesign 2016, Vol. 1, Trondheim, 10.-12.08.2016, Design Society pp. 332-341.

Kaspar, J., Choudry, S. A., Landgrebe, D., and Vielhaber, M. (2018), "Concurrent selection of material and joining technology - an initial utility-based systematic decision-making tool”, 2018 Annual IEEE International Systems Conference (SysCon), Vancouver, 23.-26.04.2018, IEEE, pp. 767-774 https://doi.org/10.1109/SYSCON.2018.8369549.

Kaspar, J., and Vielhaber, M. (2018), "Integrated cross-component lightweight and material-oriented development methodology - the embodiment design cycle”, 28th CIRP Design Conference, Nantes, 23.-25.05.2018, Vol. 70, pp. 481-486. https://doi.org/10.1016/j.procir.2018.03.001.

Kett, S. G., and Wartzack, S. (2016), "Considering emotional impressions in product design: Quality of life theory and its impact on design strategy”, 14th International Design Conference - DESIGN 2016, Dubrovnik, 16.19.05.2016, Design Society, pp. 1719-1728.

Lima-Junior, F. R., and Carpinetti, L. C. R. (2016), "A multicriteria approach based on fuzzy-QFD for choosing criteria for supplier selection”, Computers \& Industrial Engineering, Vol. 101, pp. 269-285. https://doi.org/10.1016/j.cie.2016.09.014.

Pahl, G., Beitz, W., Feldhusen, J., and Grote, K.-H. (2007), “Engineering Design”, Springer, London. https://doi.org/10.1007/978-1-84628-319-2.

Pasini, D. (2007), "Shape transformers for material and shape selection of lightweight beams", Materials \& Design, Vol. 2 No. 7, pp. 2071-2079. https://doi.org/10.1016/j.matdes.2006.05.028.

Pohlmeyer, A. E. (2012), Identifying attribute importance in early product development - exemplified by interactive technologies and age, $\mathrm{PhD}$ Thesis, Fakultät V - Verkehrs- und Maschinensysteme, TU Berlin.

Ullman, D. G. (2010), “The mechanical design process", MacGraw-Hill, New York.

Schneberger, J.-H., Luedeke, T., and Vielhaber M. (2018), “Agile Transformation and Correlation of CustomerSpecific Requirements and System-Inherent Characteristics - An Automotive Example", 28th CIRP Design Conference, Nantes, 23.-25.05.2018, Vol. 70, pp. 78-83. https://doi.org/10.1016/j.procir.2018.03.068.

Sim, W. W., and Brouse, P. S. (2014), "Empowering requirements engineering activities with personas", Conference on Systems Engineering Research (CSER) 2014, Redondo Beach, 20.-22.03.2014, Vol. 28, pp. 237-246. https://doi.org/10.1016/j.procs.2014.03.030.

Ulrich, K. T., and Eppinger, S. D. (2008), "Product Design and Development". MacGraw-Hill, New York.

Weaver, P.M., and Ashby, M. F. (1997), "Material limits for shape efficiency", Progress in Materials Science, Vol. 41, No. 1-2, pp. 61-128. https://doi.org/10.1016/S0079-6425(97)00034-0. 